\title{
UNE FINANCE RESPONSABLE À L'ĖRE DE LA MONDIALISATION ÉCONOMIQUE
}

\author{
Corinne Gendron et Gilles L. Bourque
}

Altern. économiques | « L'Économie politique»

$2003 / 2 n^{0} 18 \mid$ pages 50 à 61

ISSN 1293-6146

Article disponible en ligne à l'adresse :

https://www.cairn.inforevue-l-economie-politique-2003-2-page-50.htm

Distribution électronique Cairn.info pour Altern. économiques.

(C) Altern. économiques. Tous droits réservés pour tous pays.

La reproduction ou représentation de cet article, notamment par photocopie, n'est autorisée que dans les limites des conditions générales d'utilisation du site ou, le cas échéant, des conditions générales de la licence souscrite par votre établissement. Toute autre reproduction ou représentation, en tout ou partie, sous quelque forme et de quelque manière que ce soit, est interdite sauf accord préalable et écrit de l'éditeur, en dehors des cas prévus par la législation en vigueur en France. Il est précisé que son stockage dans une base de données est également interdit. 
[1] Nous tenons à remercier le programme INÉ, du CRSH, qui a permis de mener les recherches ayant servi de base au présent article. II s'inspire de plusieurs de nos recherches, notamment Gilles L. Bourque et Corinne Gendron, «La finance responsable : la nouvelle dynamique d'une finance plurielle ?», Economie et Solidarités $\mathrm{n}^{\circ} 2$, vol. 34 (à paraître, 2003), et Gilles L. Bourque et Daniel Simard, «Une expérience québécoise de finance responsable : la CSN et ses outils collectifs », Cadres CFDT $n^{\circ} 400$, juillet 2002.

\section{Une finance responsable à l'ère de la mondialisation économique}

\section{Corinne Gendron,}

professeure à l'Uqam (Université du Québec à Montréal), et Gilles L. Bourque,

économiste chez FondAction [1]

U MILIEU DES ANNÉES 1970, LE RÉGIME D’ACCUMULATION fordiste s'est grippé, sonnant le glas des Trente Glorieuses qui ont caractérisé la période de l'après-guerre. Jusqu'alors ancré dans le territoire national, le développement est devenu affaire de production, puis de commerce international. Impulsé par les stratégies de multinationalisation des grandes entreprises, le nouvel espace économique mondial a trouvé, dans les nouvelles technologies de communication, le terreau de son expansion et de son approfondissement. Cet espace mondial est devenu peu à peu l'élément de référence des stratégies corporatives, conduisant à une profonde recomposition du politique et des rapports entre entreprise et Etat. Cette recomposition a été d'autant plus radicale que, poussé par les nouvelles technologies de communication, le mouvement de décloisonnement, de désintermédiation et de déréglementation (communément appelé «mouvement des trois $D »)$ est venu consacrer le démantèlement du système monétaire et favoriser l'intégration d'une finance désormais mondialisée et hautement volatile. 
Les modalités de régulation de ce nouveau régime sont au cœur des controverses de notre temps. Les nouvelles instances de la mondialisation en sont à échafauder un système complexe où le libre commerce est érigé en principe légitimateur, alors que des acteurs sociaux dispersés proposent une mondialisation alternative, portée par d'autres principes, et s'engagent dans un processus d'organisation réticulaire inédit où l'action sociale prend de nouvelles formes. Ces acteurs ont ceci de particulier qu'ils ne se contentent plus de la sphère politique et sociale pour déployer leur action ; ils investissent désormais le champ autrefois suspect de l'économie. Le commerce équitable, les investissements socialement responsables, la finance solidaire et l'économie sociale ont en commun le fait d'instrumentaliser l'économie à des fins socio-politiques. Ces mouvements contestent ainsi le dogme d'une économie a-sociale purement fonctionnelle, et rendent visibles les implications politiques des postulats de l'économie traditionnelle. Ils proposent une nouvelle économie, réencastrée dans le social et inscrite au cœur d'un projet de développement librement choisi : l'économie pensée comme moyen peut être configurée en fonction de choix politiques et sociaux faits en amont.

Les innovations sociales du secteur financier sont tout à fait représentatives de cette appropriation de l'économie par ceux que nous avons convenu d'appeler les nouveaux mouvements sociaux économiques [2]. La finance socialement responsable propose en effet de nouvelles modalités de fonctionnement, tendant à rendre explicite la performance sociale des actes financiers. En conséquence, elle permet d'orienter ces actes en fonction de critères socio-économiques, donnant potentiellement corps à un modèle de développement choisi en amont du système financier lui-même.

\section{La pratique financière comme outil de mobilisation sociale}

Au cours de sa courte histoire, l'investissement éthique pratiqué par les communautés religieuses inquiètes des activités immorales telles que l'alcool et le jeu (sin stocks) a progressivement cédé le pas à un mouvement d'investissements socialement responsables préoccupés par les questions de droits humains, de droits du travail et d'environnement. Ainsi, dès les années 1960, les activistes américains du mouvement des droits civiques et contre la guerre du Vietnam rejoignent les communautés religieuses dans leurs efforts de mobilisation. A cette occasion, les critères éthiques utilisés dans la gestion financière
[2] Voir Corinne Gendron, «Emergence de nouveaux mouvements sociaux économiques", Revue Pour $\mathrm{n}^{\circ}$ 172, Paris, Grep, 2001. 
prennent un caractère de plus en plus social. Mais cette mobilisation financière conserve la même forme, celle d'un tamisage par l'application de filtres négatifs au moment du choix des placements financiers.

Dans les années 1970 et 1980, la mobilisation financière prend son envol à l'international en se concentrant sur la lutte contre l'apartheid en Afrique du Sud. Elle sort du territoire américain et se répand en Grande-Bretagne, où les liens avec l'Afrique du Sud sont évidents, puis sur le continent européen. C'est dans ce contexte que se produit un tournant important, qui débouchera sur une nouvelle forme de placement. Alors que la finance éthique se limitait à une approche punitive, de rejet de certains placements en fonction de critères moraux, un activiste américain, Leon Sullivan, propose une approche d'engagement : les placements doivent servir à changer les conduites des entreprises, particulièrement en faisant en sorte qu'elles deviennent des acteurs de réforme du régime de l'apartheid par l'application volontaire de politiques d'emploi non discriminatoires.

Ce premier pas sur le plan international a rapidement servi de modèle pour étendre le mouvement des investissements responsables à d'autres causes, en particulier à celle de l'environnement. Alors que l'on pouvait craindre que le mouvement des investisseurs responsables ne s'effondre avec la fin de l'apartheid en Afrique du Sud, celuici, au contraire, a connu un nouveau

LA FIN DES ANNÉES 1990 VOIT UNE VÉRITABLE INSTITUTIONNALISATION

DU MOUVEMENT DE L'INVESTISSEMENT RESPONSABLE, ALORS QUE LES LÉGISLATIONS RECONNAISSENT SON EXISTENCE ET QU'IL SE STRUCTURE EN INDUSTRIE. souffle avec la cause environnementale, qui s'est progressivement imposée comme le critère dominant des fonds éthiques. Au cours des années 1990, le mouvement de l'investissement responsable a poursuivi son essor, à la faveur de la diversification des thèmes de mobilisation sociale. Les conséquences de la mondialisation ont donné prise à plusieurs campagnes pour dénoncer les conditions de travail dans le Sud, notamment le travail des enfants. Parallèlement, la mondialisation financière a suscité l'émergence d'une nouvelle finance solidaire territorialisée, axée sur les besoins des collectivités, et l'apparition de nouveaux acteurs sur la scène financière, tels que les fonds de travailleurs. Dans la même foulée, on a vu des institutions financières allouer une partie de leurs actifs 
à de nouvelles institutions financières visant le développement économique et social de leur communauté. Aux préoccupations environnementales se sont ainsi ajoutées des préoccupations plus spécifiquement sociales, telles que le respect des normes internationales de travail dans les pays du Sud ou le développement des communautés en difficulté dans les pays du Nord.

A la fin des années 1990, on peut parler d'une véritable institutionnalisation du mouvement de l'investissement responsable, alors que les législations reconnaissent son existence et qu'il se structure de plus en plus en industrie. A tel point que certains analystes se demandent si l'investissement socialement responsable n'est pas en passe de se banaliser, pour devenir un simple segment de marché destiné au nouveau créneau des «investisseurs socialement responsables».

\section{Finance, éthique et responsabilité sociale}

Ce petit rappel historique permet de voir que, même si certains les confondent, il est pertinent de distinguer l'investissement éthique, auquel on peut attribuer une connotation morale, de l'investissement socialement responsable, qui évoque la dimension et les impacts sociaux de tout investissement. Tandis que le premier, qui marque les débuts du mouvement, suppose l'application de critères et de jugements moraux individuels, le second, plus présent aujourd'hui, se réfère davantage aux impacts sociaux des activités et des investissements qui les supportent. En d'autres termes, l'investissement éthique renvoie à des valeurs morales reposant sur des choix individuels particuliers, alors que l'investissement social suppose une évaluation systématique et partagée des activités et de leurs conséquences sur la société, effectuée sur la base de normes sociales largement débattues et reconnues.

La notion d'investissement, traduite du terme anglais investment, n'est en fait pas très appropriée pour analyser les transformations en cours dans les pratiques financières. Le terme anglais confond les activités de placement et celles d'investissement, que l'on distingue en français. A partir du terme unique d'investment, la littérature portant sur les investissements responsables a classé ceux-ci en trois grandes formes distinctes : l'application de filtres positifs ou négatifs au moment du choix de placement (screening ou tamisage) ; l'engagement ou l'activisme actionnarial auprès des entreprises du portefeuille de placement ; 
et l'investissement communautaire. Ces formes d'activités financières correspondent d'abord et avant tout aux pratiques de l'investissement responsable telles qu'elles ont émergé aux Etats-Unis. Or, ce classement ne tient pas compte de la distinction fondamentale entre placement et investissement, d'une part, et ignore d'autre part les pratiques financières alternatives apparues ailleurs, telles que les fonds de travailleurs au Québec ou la finance solidaire en Europe. C'est pourquoi nous proposons plutôt, comme traduction de l'expression "socially responsible investment », la notion de "finance socialement responsable » (FSR), qui intègre tout en les distinguant les aspects placement et investissement.

Cette notion permet de développer une nouvelle typologie, où la finance socialement responsable se compose de deux grands types de pratiques distinctes : les placements et les investissements. Alors que le placement se réfère à l'action de placer de l'argent, l'investissement renvoie à l'emploi de capitaux visant à accroître la production ou à améliorer le rendement. En d'autres termes, les placements concernent généralement les activités financières sur les marchés secondaires, alors que les investissements concernent des participations directes en entreprise. Mais, qu'il s'agisse de placement ou d'investissement, la finance socialement responsable peut prendre diverses formes et adopter plusieurs véhicules.

\section{Les placements}

Les deux principales formes de placements socialement responsables correspondent aux pratiques financières alternatives qui ont émergé aux Etats-Unis et qui se sont répandues presque partout où il existe des marchés organisés de capitaux : le tamisage et l'engagement (ou l'activisme actionnarial). Le tamisage se pratique par l'application de filtres positifs ou négatifs, sur la base de critères éthiques, sociaux ou environnementaux, dans le processus de choix des placements. Les filtres sont négatifs lorsqu'ils visent à exclure certaines entreprises du portefeuille de placement - par exemple des producteurs de cigarettes, d'armes ou encore des entreprises qui ont un bilan social ou environnemental déficient. A contrario, les filtres positifs visent à ajouter dans le portefeuille des entreprises qui se signalent par des pratiques exemplaires, qui apportent une contribution significative au développement durable, ou encore qui font preuve d'une gouvernance d'entreprise participative. 
L'engagement, qu'on appelle souvent l'activisme actionnarial, adopte une philosophie d'action différente, même s'il s'appuie sur des critères identiques au tamisage. La philosophie de l'engagement est de contribuer au changement des pratiques des entreprises au chapitre de leur responsabilité sociale et environnementale. Les activistes utilisent leur influence en tant qu'actionnaires ou même en tant que simples détenteurs d'obligations, dans le but d'améliorer la gouvernance et les pratiques sociales et environnementales de l'entreprise. Pour ce faire, ils engagent tout d'abord un dialogue avec la direction, afin d'obtenir des informations sur les pratiques de l'entreprise ou de persuader les dirigeants de modi-

\section{LA DISTINCTION ENTRE CAPITAL DE DÉVELOPPEMENT ET FINANCE SOLIDAIRE} SEMBLE LA PLUS APPROPRIÉE POUR SAISIR LES TENDANCES DE FOND DES INVESTISSEMENTS SOCIALEMENT RESPONSABLES.

fier certaines pratiques socialement inacceptables. Lorsque le dialogue ne donne pas de résultat, ces activistes déposent des résolutions d'actionnaires ou votent des résolutions à caractère social lors des assemblées d'actionnaires. Enfin, ces activistes iront jusqu'à la menace de désinvestissement, en liaison avec des campagnes actives de boycottage. Le désinvestissement peut ainsi être reconnu comme une troisième forme de stratégie de placement socialement responsable, puisqu'il combine tout à la fois l'engagement et le tamisage, auquel il peut aboutir si le dialogue prévu par la stratégie d'engagement échoue.

\section{Les investissements}

On peut classer les pratiques de l'investissement socialement responsable en deux grandes catégories, selon qu'il s'agit de capital-développement ou de finance solidaire. Dans ce domaine, les expériences nationales sont multiples ; chaque pays a innové, et on y observe une multitude d'expériences de services financiers cherchant à répondre à des besoins nouveaux. La distinction entre capital de développement et finance solidaire nous apparaît comme la plus appropriée pour saisir les tendances de fond des investissements socialement responsables.

Le capital développement doit être compris comme une forme de capital-risque dont le rendement anticipé sur les investissements ne vise pas tant à maximiser qu'à pérenniser l'activité économique. Comme le capital-risque, le capital développement attend un rendement par rapport aux risques encourus. Cependant, 
contrairement aux sociétés de capital-risque classiques, les fonds de capital développement ont comme objectif principal le développement économique de leur territoire, dans le but de créer de l'emploi. Ils se distinguent aussi des organismes publics par le fait qu'ils fournissent du financement, plutôt que des subventions aux entreprises.

[3] Voir Corinne Gendron, "Fonds de pension à la québécoise", Le Monde diplomatique, avril 2002 (ou à l'adresse : http://www.mondediplomatique.fr/2002/04/ GENDRON/16383).
Au Québec, l'exemple type de ce capital de développement est celui des fonds de travailleurs [3]. En retour des avantages fiscaux dont bénéficient les souscripteurs, la législation impose à ces fonds l'obligation de combiner l'objectif de rendement à des objectifs de création d'emplois, de développement économique, de participation et de formation des travailleurs. Un pourcentage important de l'actif des fonds de travailleurs doit prendre la forme de participations directes dans des PME québécoises, le reste pouvant être utilisé sous forme de placements en obligations ou en valeurs mobilières. Dans les pays de tradition anglo-saxonne, où les fonds de pension privés sont plus développés, on a vu émerger ces dernières années plusieurs formes de véhicules financiers semblables aux fonds de travailleurs. Aux Etats-Unis, on nomme ces pratiques les investissements économiquement ciblés (economically targeted investments) : ils visent à combler des lacunes de financement qui entraînent des sous-investissements dans certains secteurs. De façon générale, les promoteurs de cette finance socialement responsable sont des fonds de pension qui mutualisent les risques en plaçant une partie de leur actif dans une société d'investissement qui se donne des objectifs de création d'emploi, de développement économique communautaire, de logements abordables, d'infrastructures publiques, etc.

La finance solidaire, quant à elle, renvoie à une pluralité de véhicules d'intervention, tout en étant nécessairement gouvernée par les acteurs locaux. Elle s'inscrit le plus souvent dans la perspective du développement économique communautaire, qui se définit comme une stratégie multifonctionnelle et globale conçue et dirigée localement, dans le but de contribuer à la revitalisation et au renouvellement des économies des communautés locales. La finance solidaire tente de répondre à la difficulté, pour les collectivités en déclin et les populations aux prises avec 
le cercle vicieux de la pauvreté, d'accéder à du capital, les outils de la finance traditionnelle n'étant généralement ni disponibles ni adaptés aux besoins de ces populations. Comme la finance traditionnelle, la finance solidaire peut prendre la forme de prêts à intérêts avec ou sans garanties, mais elle passe aussi par la reconstruction du capital social des communautés. Les investissements de la finance solidaire visent évidemment des résultats quantitatifs (création d'emplois ou d'entreprises, en particulier des entreprises d'économie sociale), mais surtout qualitatifs : employabilité, perfectionnement, nouvelle culture entrepreneuriale, autocontrôle, gains d'autonomie...

Les véhicules de la finance solidaire sont diversifiés : le crédit communautaire, le microcrédit ou les cercles d'emprunt occupent le créneau des très petits prêts, en offrant du crédit et de l'accompagnement aux personnes et aux groupes qui cherchent à démarrer ou à consolider des projets générateurs de revenus autonomes. D'autre part, les Credit Unions au Canada, les caisses populaires et d'économie au Québec, les banques éthiques en Italie, les caisses solidaires et les fonds de partage en France peuvent être, dans une certaine mesure, classés comme véhicules de la finance socialement responsable, lorsqu'ils se donnent un rôle de développement social spécifique dans leur communauté d'appartenance.

\section{Une nouvelle régulation portée par des modalités inédites de l'action collective}

La finance responsable, qu'on peut également caractériser de plurielle, compte tenu de la diversité des instruments qui s'en réclament, existe et se développe parce qu'en amont, tout d'abord, des personnes s'approprient les valeurs et les principes d'une éthique autre que celle, exclusive, du profit à court terme. Mais c'est aussi parce que, en aval, cette finance répond à des besoins provenant d'entrepreneurs individuels et collectifs qui intègrent, dans leur processus de décisions, des critères relevant de l'intérêt général.

Le placement responsable permet aux investisseurs préoccupés par l'impact de leurs choix financiers de se prononcer sur les pratiques des grandes entreprises "publiques » [4]. Il corres-

[4] Entendues ici dans le sens de sociétés à capital ouvert. pond à un nouveau véhicule de pression sociale : au lieu d'utiliser des moyens de pression traditionnels, les mouvements sociaux accaparent le statut d'actionnaire pour forcer l'entreprise à tenir compte de considérations sociales, en sus de la recherche d'un 
rendement financier. Cette utilisation inédite du statut d'actionnaire pose un véritable défi au système juridique, puisque, loin d'être propriétaire de l'entreprise, l'actionnaire n'a que des droits d'investisseur vis-à-vis d'elle. En conséquence, il ne peut contester ses pratiques que dans la mesure où celles-ci présentent un risque pour son rendement.

C'est dans cette optique que, jusqu'à tout récemment, la loi sur les sociétés canadiennes par actions permettait aux entreprises de rejeter toute proposition des actionnaires ayant pour objet principal de «servir des fins générales d'ordre économique, politique, racial, religieux, social ou analogue». Amendé à la fin de l'année 2001, le régime juridique canadien permet désormais de déposer de telles propositions, à condition que ces dernières soient liées aux activités commerciales et aux affaires internes de la société. Cet amendement étend, il est vrai, le champ d'action des actionnaires, mais il faut y voir davantage une compréhension plus large des facteurs de rentabilité et de risque qu'une transformation dans la nature du droit des actionnaires vis-à-vis de l'en-

LES RÉSOLUTIONS D'ACTIONNAIRES À CARACTÈRE SOCIAL OU ENVIRONNEMENTAL,

SI ELLES NE RECUEILLENT QUE 10 À $30 \%$ DES VOTES, ONT UN IMPACT CONSIDÉRABLE SUR LES POLITIQUES DE L'ENTREPRISE. treprise. II n'en reste pas moins que, en pratique, les résolutions d'actionnaires à caractère social ou environnemental, alors même qu'elles ne recueillent que 10 à $30 \%$ des votes, ont un impact considérable sur les politiques de l'entreprise ; et cela est probablement moins dû à la menace de contrainte juridiques qu'elles représentent qu'au battage médiatique dont ces résolutions sont l'objet. Dans nombre de cas, la direction négocie avec les actionnaires activistes en vue du retrait de la proposition au moment de l'assemblée. Cette pratique a donné lieu à l'adoption de plusieurs codes de pratiques en matière de droits humains et au chapitre de l'environnement par des firmes traditionnellement réfractaires aux stratégies de responsabilité sociale. Par ailleurs, l'engagement concerne souvent les activités extraterritoriales des entreprises, qui échappent à la juridiction de leur pays d'attache. Le placement responsable se positionne ainsi comme régulateur d'agents économiques de plus en plus difficiles à saisir et à encadrer, et les campagnes d'actionnaires favorisent les comportements corporatifs socialement responsables, tout en fournissant aux parties prenantes de l'entreprise des informations essentielles à la prise de décision. 
Si les placements responsables ont l'ambition d'encadrer et de surveiller les pratiques sociales et environnementales des grandes entreprises, on pourrait dire que l'investissement responsable tente de domestiquer la finance elle-même. A l'heure où les marchés financiers interdépendants sont de plus en plus

L'INVESTISSEMENT RESPONSABLE TENTE DE DOMESTIQUER LA FINANCE ELLE-MÊME. instables et volatils, l'investissement responsable territorialise le capital en y accolant des projets concrets de développement ancrés dans les populations. Il participe notamment au renouveau de l'entrepreneuriat individuel et collectif, auquel il dédie une partie importante de ses capitaux. En finançant des projets concrets portés par les communautés locales, l'investissement responsable est un exemple probant d'instrumentalisation de l'économie à des fins sociales, qui a l'intérêt de rapprocher l'investissement de sa finalité originelle : le développement.

De façon plus générale, et qu'il s'agisse de placements ou d'investissements, la finance responsable se démarque d'une individualisation des outils financiers à laquelle a présidé la financiarisation de l'économie et l'internationalisation de la finance. Elle vient reconstruire le lien entre l'investisseur et le projet de développement, en se préoccupant de la question de la cohésion sociale. Les attentes à court terme sont reléguées au second plan, et les bénéfices ne sont plus mesurés uniquement à l'aune du rendement financier individuel. Il s'agit donc d'un capital patient, bénéficiant d'un rendement satisfaisant, mais avec une performance sociale et environnementale plus élevée sur le long terme. L'intérêt de cette finance responsable est qu'elle tend à resocialiser une économie qu'on a longtemps cherché à émanciper de son ancrage social. Peu à peu, les différents acteurs sociaux s'approprient des mécanismes, des techniques et un champ acquis à des experts du capital, pour y impulser d'autres finalités et en élargir le potentiel social.

Porteurs de nouvelles régulations, ces acteurs sociaux proposent une modalité inédite de l'action collective. Ainsi, même si l'on peut les présenter comme une troisième génération de mouvement social, les nouveaux mouvements sociaux économiques ne viennent pas tant remplacer les mouvements précédents, comme l'ont parfois interprété certains, mais plutôt ouvrir 
des champs d'intervention dans la sphère autrefois suspecte de l'économie. Les nouveaux mouvements sociaux économiques sont donc en lien, et même souvent issus des mouvements sociaux des générations précédentes.

L'acteur syndical est exemplaire à cet égard. Le changement d'orientation du mouvement syndical des années 1980, lorsque la confrontation a cédé le pas à une concertation conflictuelle, a ouvert la porte à de nouvelles stratégies pour défendre l'intérêt collectif des travailleurs. Comme l'affirmait le secrétaire général du Réseau international de syndicats, l'Union Network International (UNI), Phil Jennings : "Nous sommes constamment en quête de nouvelles méthodes de pression pour amener les entreprises à assumer leur responsabilité sociale. » Ainsi, pour influencer le processus de mondialisation dans le sens du développement durable et du respect des droits humains et sociaux, le mouvement syndical s'est mobilisé sur plusieurs fronts. Il s'est rendu compte que la finance responsable pouvait représenter l'un des fers de lance de l'intervention syndicale, là où la mondialisation a les répercussions les plus immédiates et les plus néfastes pour l'ensemble des travailleurs.

La Confédération internationale des syndicats libres (CISL) s'est positionnée sur le sujet et explore plusieurs voies d'intervention. Considérant que le mouvement syndical international est le mieux placé pour influencer le débat et participer à l'émergence de nouvelles pratiques dans ce domaine, la CISL, en partenariat avec les Secrétariats professionnels internationaux (SPI) et la Commission syndicale consultative (TUAC) auprès de l'OCDE, a créé le Comité sur la coopération internationale relative au capital des travailleurs. L'objectif principal de ce Comité est de faire entendre, au niveau international, la voix du capital des travailleurs, de manière à pouvoir influencer les pratiques des entreprises. Le Comité veut faire de l'engagement corporatif le pilier de son intervention. En particulier dans le domaine du travail, son engagement visera à ce que les entreprises adhèrent aux principes contenus dans la Déclaration de l'Organisation internationale du travail (OIT). La Déclaration relative aux droits fondamentaux du travail stipule en

[5] Ces conventions traitent de la liberté syndicale, de l'abolition du travail forcé, de l'égalité, et de l'élimination du travail des enfants. effet que l'ensemble des membres de l'OIT ont l'obligation de respecter, promouvoir et réaliser les droits contenus dans les conventions fondamentales de l'Organisation [5], même s'ils n'ont pas ratifié ces conventions. 
A la lumière de ce qui précède, on peut voir que le mouvement syndical est sans contredit un acteur omniprésent au sein des nouveaux mouvements sociaux économiques. Par ailleurs, le cas syndical illustre bien comment la sphère financière tout comme la sphère plus générale de l'économie sont désormais investies par des acteurs sociaux soucieux de les configurer en fonction d'autres valeurs que celles qui y ont présidé jusqu'à maintenant. Les nouvelles pratiques, tout comme les nouvelles institutions financières auxquelles elles ont donné naissance, ancrées dans des mouvements sociaux divers tels que les syndicats, les communautés ou le mouvement écologiste, sont l'antithèse de la finance spéculative. En agissant sur le moyen et le long termes et en intégrant des préoccupations sociales dans leurs processus de décision, elles combinent l'économique et le social, le local et le global. Ces mouvements sociaux réinventent l'économie et la finance ; ils les resocialisent en les reléguant au rang d'outil

\section{LE MOUVEMENT SYNDICAL}

S'EST RENDU COMPTE QUE LA FINANCE RESPONSABLE POUVAIT REPRÉSENTER L'UN DES FERS DE LANCE DE SES INTERVENTIONS. et de moyen, et en leur donnant une finalité qui ne dépend plus de la véracité des théories économiques pour être atteinte. La montée de l'éthique peut donc s'interpréter dans le cadre de la réorganisation des pôles de régulation, portée par une société civile qui réinvente l'action collective, au point de donner forme à une régulation «de troisième type » entre l’Etat et le marché. 